\title{
Montage Serial Sectioning: Some Finer Aspects of Practice
}

\author{
Ashok Gurumurthy • Arun M. Gokhale • \\ Anshul Godha $\cdot$ Manny Gonzales
}

Received: 29 July 2013/Accepted: 10 October 2013/Published online: 31 October 2013

(C) Springer Science+Business Media New York and ASM International 2013

\begin{abstract}
Serial sectioning as a probe of topological properties of solids has become increasingly important for quantitative materials characterization and provides accurate inputs for microstructure simulations. In recent years, large-area montage serial sectioning has gained traction, due to the high resolution of micrographs it produces and the large volumes it samples. But some finer aspects of the practice dealing with error detection and correction-necessary for accurate reconstruction of a microstructural volume-have not received much attention. This article describes the tools and techniques we developed to (1) correct shading in tiles that make up a montage and (2) detect metallographic errors in serial sectioning. These tools help reconstruct the most accurate three-dimensional microstructures for use in investigating the micromechanics of materials under a variety of conditions. They also help ensure accuracy in stereological measurements, in reconstruction of constituent morphologies for characterization or simulation, and in other applications of montage serial sectioning.
\end{abstract}

Keywords Montage serial sectioning - Shading correction · Affine transform - Three-dimensional microstructure

This article is an invited paper selected from presentations at the 2013 Quantitative Metallography Conference and Exposition, held April 4-5, 2013, in San Antonio, TX, and has been expanded from the original presentation.

A. Gurumurthy $(\bowtie) \cdot$ A. M. Gokhale · A. Godha · M. Gonzales School of Materials Science and Engineering, Georgia Institute of Technology, Atlanta, GA 30332-0245, USA

e-mail: ashokg@gatech.edu

\section{Introduction}

Metallographers, biologists, mineralogists, and other specialists have long used serial sectioning [1-3], a destructive characterization tool, to measure those properties of threedimensional (3-D) solids that cannot be measured from two-dimensional (2-D) sections, namely topological properties [4, 5]. It is becoming increasingly important for quantitative materials characterization and analysis and for providing inputs to 3-D microstructure simulations [6, 7]. Modern microscopes have made it possible to image large areas of a sample automatically and quickly, leading to the development of montage serial sectioning [4], which can sample large volumes of microstructure. There are, however, aspects of montage serial sectioning that deserve closer attention than they have received, if it is to be wielded as a high-precision tool.

Serial sectioning is susceptible to imaging and metallographic errors. In this article, we describe the tools and techniques we developed to (1) correct shading, which is an artifactual variation in the intensity across a field of view (FOV), in montaged section images, and to (2) quantitatively estimate two types of metallographic errors in the serial sectioning: variable inter-section distance, and non-parallelism between sections. Correcting for the metallographic errors, after obtaining section images, is quite difficult, and we shall discuss some methods to do so in a forthcoming article.

We should like to synopsize here the particular flavor of montage serial sectioning that we refer to everywhere else, to provide some background for the coming sections. Tewari and Gokhale [4] provide a detailed description of the same process, as do Singh et al. [5]. Serial sectioning reconstructs a microstructural volume of a sample by metallographically preparing and imaging several sections 

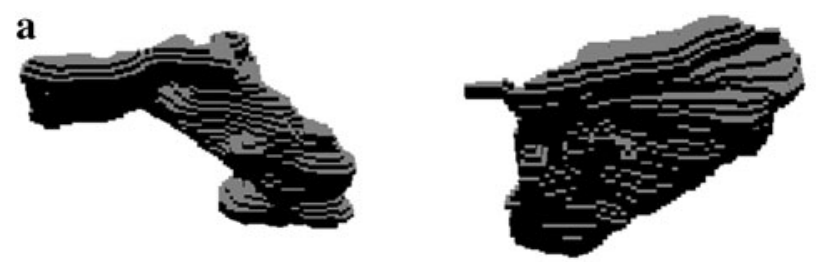

$15.5 \mu \mathrm{m}$

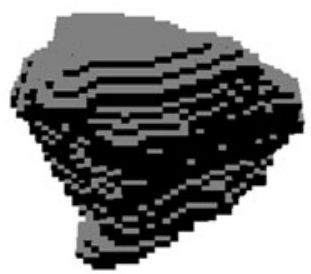

$11.1 \mu \mathrm{m}$

$10.4 \mu \mathrm{m}$

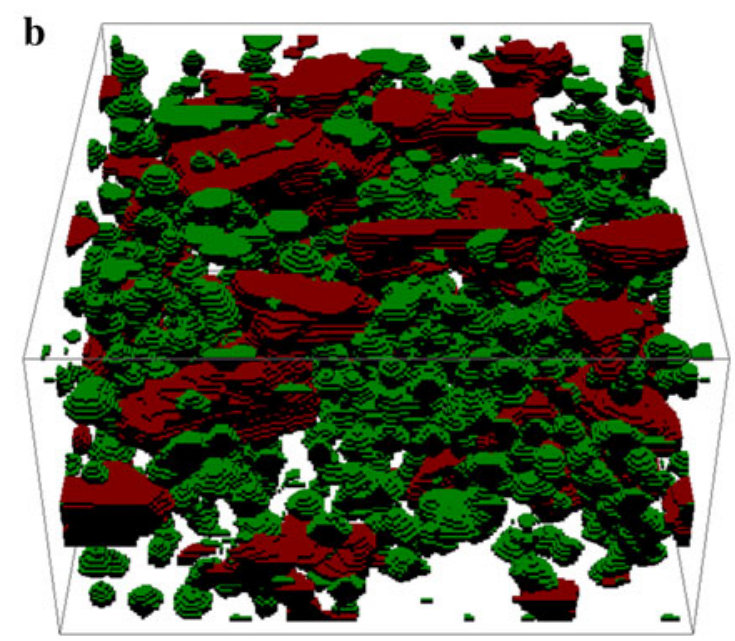

Fig. 1 Reconstruction and simulation using montage serial sectioning: (a) some reconstructed elemental sponge titanium particles obtained from serial sections through an aluminum-titanium binary powder mixture compact (sizes shown are volume-equivalent sphere diameters), and (b) a $60 \mu \mathrm{m} \times 60 \mu \mathrm{m} \times 60 \mu \mathrm{m}$ simulated 3D micrograph of composition $\mathrm{Al}-10 \% \mathrm{Ti}-10 \% \mathrm{~B}$ by volume, where the titanium particles (red) are inputs, alongside simulated boron particles (green), and aluminum (the rest of the space)

through the sample. When the images are montages of single FOVs (or tiles), we term the process montage serial sectioning, after Tewari and Gokhale [4]. Sections are typically designed to be equidistant and parallel to one another. Because polishing a sample actually abrades the surface, removes material, and exposes a fresh surface, it serves as the tool to expose the desired sections through the volume in succession.

Careful control of the process can ensure that the intersection spacing is uniform or nearly so, and that the planes of the sections are parallel to one another or nearly so. The images of the sections need to be aligned (or registered, in image-processing parlance) to be useful in reconstruction.
This means that an $(x, y)$ position on two successive section images must refer to positions exactly one below the other in the 3-D volume. Therefore, some guiding fixed points on the sections are necessary. Indents made with microindentation hardness testers are ideal for the job, because the diamond indenter precisely marks its apex below the surface of the sample. The indents may need to be renewed every few sections.

Figure 1 illustrates one possible use of such serial sectioning in microstructure simulation: we imaged titanium particles (AEE, sponge powder, $<20 \mu \mathrm{m}$ ) by montage serial sectioning of a green compact of a mixture of elemental titanium and aluminum powders. The images made up a library of particles, for use in simulation of realistic 3-D microstructures; for more details see [7], or the similar work of Singh et al. [8]. The real titanium particle images and simulated boron particle images-both 3-D-were then inputs for the simulation of the microstructure of an $\mathrm{Al}-10 \% \mathrm{Ti}-10 \% \mathrm{~B}$ (by volume) powder-mixture compact. If a simulated microstructure is to be realistic, it must have realistic particle morphologies, which it is not generally possible to obtain by other means than serial sectioning. The details of the microstructure simulation are not relevant here; the purpose is only to point out the utility of montage serial sectioning.

\section{Shading Correction in Tiles}

The illumination in a microscope is typically not uniform over the area of the sample captured in a single FOV. This causes patches (any relatively homogeneous contiguous region of the sample surface-not a technical term) in the sample that have the same reflectivity and orientation to be recorded at different grayscale intensities, depending on where within the FOV they happen to lie. The problem of variation in recorded intensities is exacerbated at low magnifications and when the FOV is large. The non-uniform intensity is usually called shading and is a common artifact of imaging systems.

It is a common occurrence, even in published micrographs and microscope images from other disciplines, to see stitched montages that show the seams, which are the result of a lighter region from one tile being placed right next to a darker region from another, contiguous, tile. It is unnecessary to cite examples, because they abound, as anyone can verify. See Fig. 2(a) for the example we are using to demonstrate our correction algorithm. The arrow points at one of the points of intersection between horizontal and vertical seams. The visible seams may strike one as merely an eyesore, with no other consequences. But the problem is neither merely esthetic nor confined to montages. 

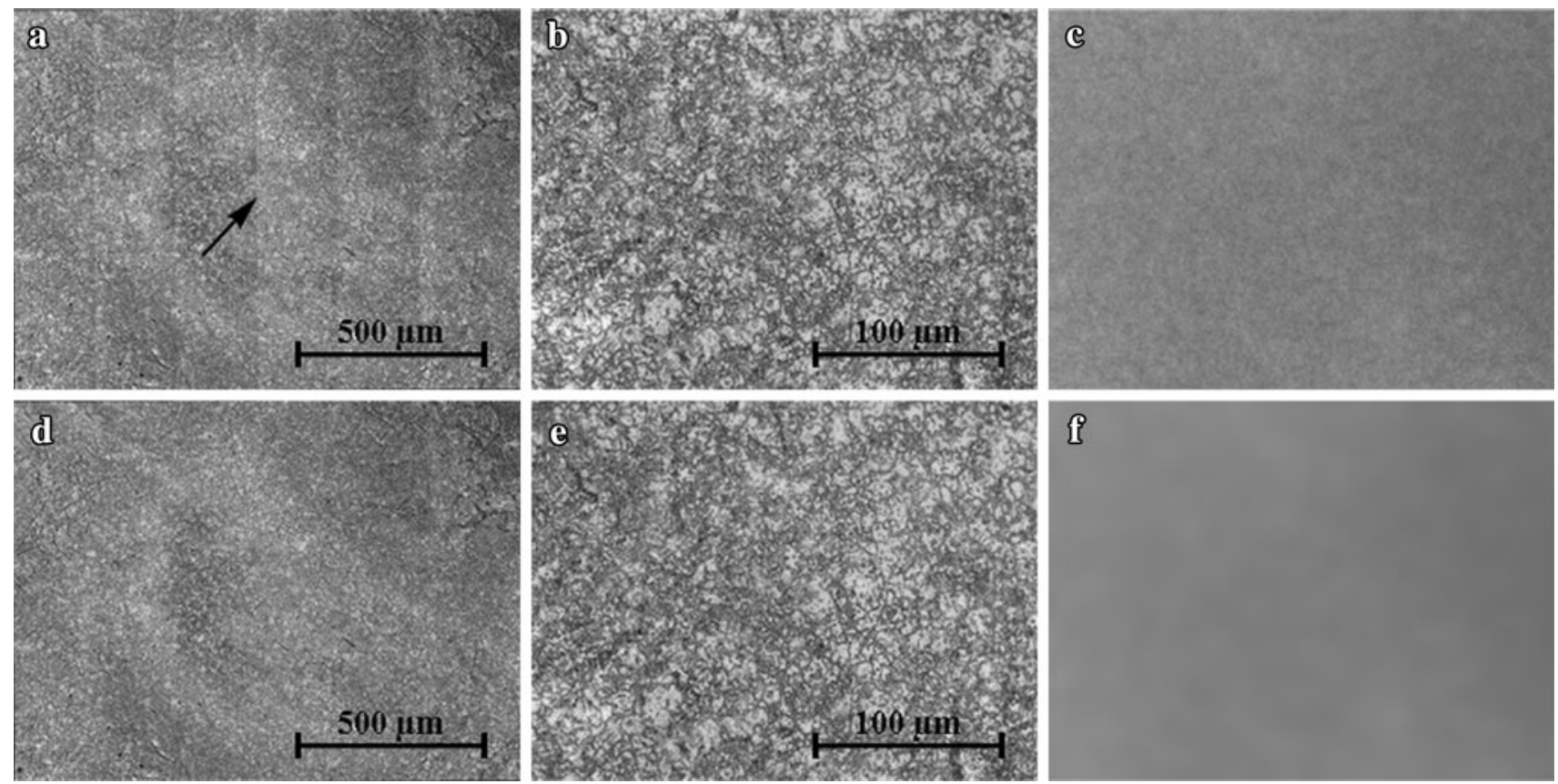

Fig. 2 Shading correction in a challenging montage micrograph of a dual-phase steel etched with nital and sodium metabisulfite, where the light regions are ferrite and the dark regions, martensite: (a) a montage with $6 \times 6$ tiles, as captured; (b) one FOV from the montage; (c) the calculated $f(x, y)$ for the montage, normalized;

Segmentation of grayscale images goes awry when identical patches of a sample surface do not record identical intensities. Image segmentation usually relies on illumination's being uniform over the entire image, producing a global image histogram that does not differ significantly from the histogram of any local region that has the same proportions of the various phases as the global average. The generalization may not hold for some segmentation algorithms that rely on local contrasts rather than global image histograms, but these algorithms apply to special cases of images, and their use is not widespread. When tiles are not stitched together, non-uniform illumination may escape notice during image capture, but it surely shows up during subsequent image processing.

The problem of shading has received a lot of attention over the years (e.g., [9-11]), leading to the development of many methods, retrospective and prospective, that try to correct it. In the comparison of various such methods by Tomaževič et al. [10], an entropy-minimization method [11] proved to be the best general-purpose corrector of shading in a single FOV. All the literature on shading correction that we are aware of deals with single FOVs. In montages, there is considerably more information present about the illumination pattern of the microscope, because the same shading pattern is present in every tile of the montage. The existing methods to correct shading fail to leverage the extra information; our method leverages it. (d) the captured montage with shading correction applied; (e) the tile in (b) with shading correction applied; (f) $f(x, y)$ after convolution with a $101 \times 101$ Gaussian kernel with $\sigma=50$ (in pixels, the tiles are 1388 wide $\times 1040$ high)

Microscopes try to correct for shading prospectively by letting the user apply a multiplicative filter to every tile: operators pick a (live) calibration image that they believe represents the illumination pattern of the light source and that can then be divided from subsequently captured images. The calibration image chosen is typically a defocused image of a relatively featureless area of the sample. How the operator can accurately assess what is a good calibration image is beyond us. For example, it is far from obvious what is the non-uniform illumination pattern in Fig. 2(b). In our experience, this prospective "correction" is usually an abysmal failure.

Fortunately, our method can always retrospectively correct for the non-uniform illumination in montages, to arbitrary precision (limited, however, by the stability of the light source, constraints imposed by the computer hardware, and the operator's patience), using image processing-sometimes easily and sometimes only with great extra effort. We shall first describe the archetypal procedure and follow it up with some variations.

\section{The Basic Method}

Let the montage be composed of $N$ tiles, $\left\{I_{k}: 1 \leq k \leq N\right\}$, and have pixels indexed by $(i, j)$. Each tile has width $W$ and height $H . I_{k}$ represents the tile as it should appear under uniform illumination of a certain intensity, and $I_{k}^{\prime}$ represents the tile as it actually appears. We can write 
$I_{k}^{\prime}(x, y)=I_{k}(x, y) f(x, y)$,

where $f(x, y)$, which we shall call the shading function, is due to the non-uniform illumination. ${ }^{1}$ Clearly, Eq. 1 only defines $f(x, y)$ to within a constant factor, so for now we assume that $f(x, y)$ is such that $I_{k}(x, y)$ and $I_{k}^{\prime}(x, y)$ have the same average brightness and postpone a quantitative statement of the assumption.

As one runs through the whole montage tile by tile, at any chosen location $(x, y)$, one expects to see different phases (or more generally, different patches, as in the terminology of the section above). If the montage consists of enough tiles, the frequency distribution of the different phases or patches occurring at $(x, y)$ in the tile will be roughly the same. Convergence to identity of frequency distributions in the limit $N \rightarrow \infty$ (both rows and columns growing) will occur whenever the microstructure does not have rigid periodicities in both $x$ - and $y$-directions with a period equal to (or a rational multiple of) that of the sampling effected by montaging. Convergence will usually be slow when there are large features, relative to the tiles, present in the microstructure.

There are several ways to solve for $f(x, y)$, not necessarily leading to the same final expression, that are strictly correct under the assumptions stated. Our preferred solution, which has given us satisfactory results, takes the logarithm of both sides and sums over $k$ :

$\sum_{k} \ln I_{k}^{\prime}(x, y)=\sum_{k} I_{k}(x, y)+N \ln f(x, y)$.

Under the foregoing assumption of large $N, \sum_{k} \ln I_{k}(x, y)$ is independent of $x$ and $y$, so that

$\sum_{k} \ln I_{k}^{\prime}(x, y) \approx N\langle\ln I\rangle+N \ln f(x, y)$

Rearranging and simplifying gives an expression for $f(x, y)$ :

$f(x, y) \approx \exp \left[\frac{1}{N} \sum_{k} \ln I_{k}^{\prime}(x, y)-\langle\ln I\rangle\right]$

We are now ready to quantify the assumption that $I_{k}$ and $I_{k}^{\prime}$ have the same average brightness: we equate their geometric means. With that, a formula for the shading function $f(x, y)$ emerges that makes use only of the observed tiles $I_{k}$ :

\footnotetext{
${ }^{1}$ A change in the brightness of a light source changes the intensity of every pixel by a constant factor. This is true both in theory and in practice for properly functioning, well-calibrated cameras. This fact motivates the form of the defining equation for $f(x, y)$.
}

$f(x, y) \approx \exp \left[\frac{1}{N} \sum_{k} \ln I_{k}^{\prime}(x, y)-\left\langle\ln I_{k}\right\rangle\right]$.

It is then easy to estimate the true tiles $\left\{I_{k}^{\prime \prime}\right\}$ (recall that $I_{k}^{\prime \prime} \rightarrow I_{k}$ as $\left.N \rightarrow \infty\right)$ :

$I_{k}^{\prime \prime}(x, y)=\frac{I^{\prime}(x, y)}{f(x, y)}$.

\section{Variations}

The basic method above will not produce satisfactory results if the montage is small or if there are large features in the microstructures, compared with the tiles. Very dark features could also cause problems. A simple test that will indicate whether a shading function calculated using Eq. 2 will correct the shading consists of examining $f(x, y)$ itself as an image, with appropriate normalization (see Fig. 2c, f, for example). If it has relatively smooth gradients such as a microscope's illumination would have, then it is likely to provide good approximations $\left\{I_{k}^{\prime \prime}\right\}$. If it shows sharp features, then $f(x, y)$ must be modified before use. The following are variations on the basic method that ensure that the algorithm always produces an acceptable corrected set $\left\{I_{k}^{\prime \prime}\right\}$.

(1) Since pixels close to each other are likely to have similar shading values (because the true shading function only has low gradients), a simple expedient is always to apply a mean filter to $f(x, y)$ before use in Eq. 3. This ensures that the errors in the estimate of $f(x, y)$ are smoothed out. The mean filter is a standard image-processing filter with parameter $r$ which produces an output image in which every pixel's intensity is the arithmetic mean of pixel intensities in an $r$-neighborhood of the corresponding pixel in the input image. Any blurring filter is an acceptable alternative to the mean filter, including the Gaussian filter (as in the example of Fig. 2) and the median filter.

(2) If basic filtering proves insufficient, the reason probably is that there are not enough tiles to keep sampling error low. Then it is best to capture extra tiles outside the region of interest, purely for the purpose of calculating $f(x, y)$, to be discarded after shading correction. If there is not extra space in the sample for additional tiles, then approximately the same region of the sample can be imaged for the extra tiles as many times as needed, but with shifted starting positions. Though the procedure is tedious, it is unlikely to need to be repeated for a given lens and a given type of sample. Using shifted montages also effectively overcomes the impediment constituted by 
strongly periodic microstructures, so long as the offsets are randomly chosen.

Figure 2 demonstrates the use of this algorithm on the micrograph of a dual-phase steel etched with nital and sodium metabisulfite. We deliberately use a relatively small montage to showcase the power of the simple algorithm. In the figure, parts (a) and (d) show the $6 \times 6$ montages before and after shading correction; (b) and (e) show a single FOV before and after shading correction; and (c) and (f) show estimates of $f(x, y)$ before and after smoothing. Note that the shading correction does not distort any features in the micrograph, and that the existence of shading is barely detectable by looking only at one FOV.

\section{Error Estimation in Serial Sectioning}

Reconstruction of the sampled volume from individual sections is easiest when the sections are parallel to one another and the inter-section distance between any two successive sections is the same throughout. Good metallographic practice seeks to minimize deviations from these two desirable conditions, but there are always errors, however small.

Mechanical vibrations can cause the polishing-machine head to tilt the sample, thus exposing a surface of the sample to the wheel that is not strictly parallel to the previous surface imaged. The resulting error we term the tilt error, defined as the angle by which any section needs to be rotated about an axis lying in its plane to bring it to the same orientation as a reference plane, which is defined as the plane perpendicular to the z-axis, taken to be the intended "depth" direction. Rotations about the axis perpendicular to the plane are easily corrected by most imaging software, as we shall discuss in a moment.

The other type of error, viz. deviation from uniform inter-section spacing, we term the misspacing error. It occurs because the material removal depends on the polishing compound's (usually a paste or a suspension) removing equal amounts of material in every cycle of polishing steps used between sections. That is very hard to ensure. The polishing cloth at the start of every cycle may not be exactly in the same condition (owing, e.g., to leftover abrasive particles or debris, or gradual tearing of the fabric); the polisher, being a mechanical system, shows variation in behavior from one cycle to the next; and when human operators are involved, they cannot be expected to maintain perfect uniformity in their handling of the machine and the suspension.

It is easy to miss and tempting to ignore these errors in the practice of montage serial sectioning and reconstruction, because reconstructed volumes do not necessarily show humanly detectable signs of these errors. But for accurate reconstruction, quantitative analysis of both types of errors is necessary and highly useful. We develop an accurate general-purpose error analysis for the first type below and discuss the second type of error in the section "Estimating Misspacing Errors."

\section{Calculating Tilt Errors}

Whenever the imager is an inverted microscope, the tilt errors of the sections can be accurately calculated. The calculation exploits the fact that the sample (embedded in the mount) rests on its prepared surface on the microscope stage, so that the image captured is the true image of the prepared surface of the sample, not a projection. Consequently, whenever there is a tilt error, the dimensions of the sample are larger in the image (only very slightly, for small tilt errors) compared with those in a reference image.

A schematic of serial sections is helpful: Fig. 3 shows a microstructural volume (a) sectioned and imaged perfectly, (b) sectioned perfectly but imaged with slight rotations, and (c) sectioned and imaged imperfectly. Only the third case involves a tilt error. The gray area in each section represents the exact area captured in the image, and the thick black lines represent the columns on which the indent apexes lie. As in the section above, a reference (section) image is an image of a section that is parallel to the reference plane and therefore perpendicular to the thick black lines running through each volume. It should be clear from the illustration why a tilt error dilates dimensions within the image.

To understand the quantitative error analysis that we describe below, it is necessary to examine how 3-D images are usually reconstructed. After every section through the sample has been metallographically prepared, an image is obtained (usually a montage of tiles) of a rectangular area of the sample demarcated by indents. The coordinates of any particular indent in the image are different in the different section images. The section images are then aligned by giving an imaging-processing application (usually integrated with the microscopy software) the positions of the three indent apexes in every section image and having it transform the images into alignment.

Such alignability depends on its being possible to bring any three ordered noncollinear points on a plane into coincidence with any other such set of points. In general, such a transform is an affine transform, a linear transformation of point coordinates, which has the property that any line (or subset of points thereon) so transformed remains a line (or a set of collinear points). 





Fig. 3 Sections in a volume: (a) perfect parallel sectioning, (b) perfect sectioning with some rotation during imaging, and (c) imperfect sectioning causing tilt errors (the four thick vertical lines inside the

Typically, angles between lines change upon affine transformation. ${ }^{2}$

The affine transform, in general, transforms a point with coordinates $(x, y)$ to $\left(x^{\prime}, y^{\prime}\right)$ according to the equation

$$
\left(\begin{array}{lll}
x^{\prime} & y^{\prime} & 1
\end{array}\right)=\left(\begin{array}{lll}
x^{\prime} & y^{\prime} & 1
\end{array}\right)\left(\begin{array}{ccc}
a_{1} & a_{2} & 0 \\
a_{3} & a_{4} & 0 \\
t_{x} & t_{y} & 1
\end{array}\right)
$$

Note that the formulation of the transform above includes the possibility of translation of the points, as well as rotation and shear. The last row of the transformation matrix, $\left(\begin{array}{lll}t_{x} & t_{y} & 1\end{array}\right)$, contains the elements for translation, and the other four variable elements relate to the shear and rotation. It is unnecessary to attempt an expression of the latter group in terms of Euler angles of rotation, because we are not interested in fully defining the 3-D rotation of the section that the transformation matrix represents, as will become clear in a moment. This matrix can be readily calculated by solving for the six independent variables in the linear equations that the matrix equation represents, using the known indent positions in the two sections to fill in the coefficients. ${ }^{3}$

\footnotetext{
2 Although it appears wrong at first to allow such shearing to occur when section images are aligned, the affine transform remains the best way to align indent apexes. The reason is that when shearing occurs, it is because simple translation and rotation were not enough to ensure coincidence of the three align points, implying tilt error. Allowing the shearing is a first-order correction of the tilt error, and there is not enough information to make possible higher-order corrections. The correction is first-order in that if features in the volume are strictly infinite cylinders, with axes perpendicular to the section plane (with arbitrary shape in the section), the affine transform fully corrects for the tilt error

${ }^{3}$ Using our error analysis requires a calculation of this matrix, so that some image-processing applications, which hide this step from the user, are unsuitable. An added advantage of aligning sections "manually" (i.e., taking control of some of the steps) is that more than three indents can be used to calculate the transformation matrix, through a least-squares fit. The least-squares approach compensates
}

volume in each of $(\mathbf{a}-\mathbf{c})$ representing the columns on which the four apexes of the guiding indents lie)

Letting the tilt error of a section under consideration be $\theta$, we can write

$\cos \theta=\frac{1}{\left|\begin{array}{ll}a_{1} & a_{2} \\ a_{3} & a_{4}\end{array}\right|}$

We should like to comment on the result before we prove it. The right-hand side of the equation above answers only to the shear part of the transform, no matter how much it rotates in plane. It should be apparent by now that it is not important to ensure while imaging each section that the sample rests in the same orientation on the microscope stage. Even if it were rotated by an arbitrary angle, the aligning software could fully compensate for it, so long as all indents are included in the captured image with reasonable padding all around. The effect of such rotation on the transformation matrix in Eq. 4 is to change the variable elements while keeping constant the determinant in Eq. 5.

Equation 5 only provides an ex post facto error estimate for the section and cannot rectify it. Nevertheless, it can guide further steps by showing up large tilt errors in time.

Turning now to the proof of the equation, any section may be thought of as a reference section that has acquired a tilt error; the reference plane, in doing so, must rotate about an axis in its plane, by definition. The effect of the rotation will be to dilate lengths perpendicular to the axis by a constant multiple $k(k>1)$, while keeping unchanged the lengths along the rotation direction. The area of any region in the reference plane changes during the rotation from $A$ to $A^{\prime}=k A$. The constant $k$ is fixed by the angle of rotation, so it does not matter what region's area $A$ represents.

Let us compute $A$ and $A^{\prime}$ for a triangle with vertices $\left(x_{1}, y_{1}\right),\left(x_{2}, y_{2}\right)$, and $\left(x_{3}, y_{3}\right)$. From elementary coordinate geometry, the area of this triangle is

Footnote 3 continued

partly for small errors in the stitching of the montage and small errors in the location of the indent apexes. 
$A=\frac{1}{2}\left[x_{3}\left(y_{1}-y_{2}\right)+x_{1}\left(y_{2}-y_{3}\right)+x_{2}\left(y_{3}-y_{1}\right)\right]$.

The triangle transforms under the general affine transform to that occupying the vertices $\left(a_{1} x_{1}+a_{3} y_{1}+t_{x}, a_{2} x_{1}+\right.$ $\left.a_{4} y_{1}+t_{y}\right), \quad\left(a_{1} x_{2}+a_{3} y_{2}+t_{x}, a_{2} x_{2}+a_{4} y_{2}+t_{y}\right), \quad$ and $\left(a_{1} x_{3}+a_{3} y_{3}+t_{x}, a_{2} x_{3}+a_{4} y_{3}+t_{y}\right)$.

Its area, by the same formula, is

$$
\begin{aligned}
A^{\prime}= & \frac{1}{2}\left(a_{2} a_{3} x_{2} y_{1}-a_{1} a_{4} x_{2} y_{1}-a_{2} a_{3} x_{3} y_{1}\right. \\
& +a_{1} a_{4} x_{3} y_{1}-a_{2} a_{3} x_{1} y_{2}+a_{1} a_{4} x_{1} y_{2}+a_{2} a_{3} x_{3} y_{2} \\
& -a_{1} a_{4} x_{3} y_{2}+a_{2} a_{3} x_{1} y_{3}-a_{1} a_{4} x_{1} y_{3}-a_{2} a_{3} x_{2} y_{3} \\
& \left.+a_{1} a_{4} x_{2} y_{3}\right)=\frac{1}{2}\left(a_{2} a_{3}-a_{1} a_{4}\right)\left[x_{3}\left(y_{2}-y_{1}\right)\right. \\
& \left.+x_{2}\left(y_{1}-y_{3}\right)+x_{1}\left(y_{3}-y_{2}\right)\right] .
\end{aligned}
$$

Setting $A^{\prime}=k A$, we obtain $a_{1} a_{4}-a_{2} a_{3}=\left|\begin{array}{ll}a_{1} & a_{2} \\ a_{3} & a_{4}\end{array}\right|$. Because $A$ is also the projected area of the triangle,

$A=A^{\prime} \cos \theta$. The result (Eq. 5) follows.

\section{Estimating Misspacing Errors}

The only general-purpose method of estimating misspacing errors-as best we know-is to introduce a lot of redundancy in the calculation of spacing. In our experience, measuring the size of the indents in any one section, for a wide variety of materials, is susceptible to large errors. If many indents are placed all around the area of interest, the spacing estimate from the different indents can be averaged for an accurate estimate of spacing. Thus do deviations from the desired spacing reveal themselves.

There exists an alternative method in one special case: if (1) there is a phase in the microstructure that is not fully connected and consists of discrete particles; (2) the inter-section spacing is much smaller than the typical $z$-ferret diameter of such particles in the microstructure; and (3) the microstructure does not have a gradient. Precisely: no statistical property of the microstructure varies with the $z$-coordinate. ${ }^{4}$

In this special case, observing the change in the area of the particles from one section to the next helps identify any misspacings. Let $a_{i}$ be the area of particle $i$ in one section, which changes to $a_{i}^{\prime}$ in the next section. The mean \langle|$a_{i}-$ $a_{i}^{\prime}|\rangle$ over all particles should be more or less constant over the set of sections if enough particles are sampled. If the mean is not approximately constant, it suggests a deviation from uniform inter-section spacing.

\footnotetext{
${ }^{4}$ It is hard to state with mathematical precision the requirements that must be met. We describe why the method works after describing what it is, and metallographers should use their judgment to decide whether the method applies to their chosen experimental conditions.
}

The method works because the area of intersection at a location between closely spaced sections may be approximated to the first two terms of its power series. That makes the change in area proportional to the distance from one section, with the proportionality constant dependent on various characteristics of the particle and on the position where the sectioning plane intersects the particle. The distribution of such characteristics encountered by a sectioning plane is the same from one section to another under the assumptions stated; therefore $\left\langle\left|a_{i}-a_{i}^{\prime}\right|\right\rangle$ is a good estimate of the inter-section distance multiplied by a microstructure-dependent constant. Other extensive characteristics of the region of intersection could have been used in place of the area, but areas can be calculated conveniently and accurately from digital images, which makes it a natural choice.

It is instructive to analyze the method mathematically for simple particle shapes, such as a frustum with arbitrary base shape and a sphere. For the frustum, letting $z$ denote the distance from the apex, the area can be written as $k_{1} z^{2}$. The change in area over a $\Delta z$ change in $z$, where $\Delta z$ is the inter-section distance, is $2 k_{1} z \Delta z$ (ignoring the $\Delta z^{2}$ term), proportional to $\Delta z$.

The sphere of radius $r$ has a section area $\pi\left(r^{2}-(z-r)^{2}\right)$, where $z$ is the distance from a point on the surface along a diameter. The difference in area between sections spaced $\Delta z$ apart is $\pi(-\Delta z+2 r-2 z)$. Averaging this difference over the range of possible $z$ values, we obtain \langle|$a_{i}-$ $a_{i}^{\prime}|\rangle=\pi \Delta z(\Delta z-2 r)^{2} /(4 r-2 \Delta z)$. Because $\Delta z$ is much smaller than $r$, the expression is approximately proportional to $\Delta z$.

\section{Summary and Conclusions}

- We described a general-purpose algorithm for retrospective shading correction in montage section images that works very well for most reasonably large montages. When combined with extra imaging steps, it achieves arbitrary precision.

- We developed a quantitative analysis for two common metallographic errors in serial sectioning: tilt and misspacing. The analysis produces accurate estimates of tilt errors generally; it provides a reasonable estimate of misspacing errors in some special cases, for use in conjunction with direct measurements of indent-size changes.

- These error correction and error tracking methods make the practice of montage serial sectioning more accurate, so that it can provide reliable measurements of microstructural properties and accurate inputs to microstructure simulations.

\section{References}

1. G. Gibbs, D. Graham, D. Tomlin, Diffusion in titanium and titanium-niobium alloys. Philos. Mag. 8(92), 1269-1282 (1963) 
2. F. Rhines, K. Craig, D. Rousse, Measurement of average grain volume and certain topological parameters by serial section analysis. Metall. Trans. A 7(11), 1729-1734 (1976)

3. D.L. DeGiusti, L. Ezman, Two methods for serial sectioning of arthropods and insects. Trans. Am. Microsc. Soc. 74(2), 197-201 (1955)

4. A. Tewari, A.M. Gokhale, Application of three-dimensional digital image processing for reconstruction of microstructural volume from serial sections. Mater. Charact. 44(3), 259-269 (2000)

5. H. Singh, A. Gokhale, A. Tewari, S. Zhang, Y. Mao, Threedimensional visualization and quantitative characterization of primary silicon particles in an Al-Si base alloy. Scr. Mater. 61(4), 441-444 (2009)

6. P. Louis, A. Gokhale, Computer simulation of spatial arrangement and connectivity of particles in three-dimensional microstructure: application to model electrical conductivity of polymer matrix composite. Acta Mater. 44(4), 1519-1528 (1996)
7. M. Gonzales, A. Gurumurthy, A. Gokhale, N. Thadhani, Mesoscale simulations of strain-induced reaction mechanisms in Ti/Al/ B heterogeneous systems. AIP Conf. Proc. 1426, 1659-1662 (2012)

8. H. Singh, A. Gokhale, A. Sreeranganathan, Y. Mao, S. Lieberman, S. Tamirisakandala, Computer simulations of "realistic" partially anisotropic microstructures statistically similar to real microstructures. Comput. Mater. Sci. 44(4), 1050-1055 (2009)

9. M.L. Schultz, L.E. Lipkin, M.J. Wade, P.F. Lemkin, G.M. Carman, High resolution shading correction. J. Histochem. Cytochem. 22(7), 751-754 (1974)

10. D. Tomaževič, B. Likar, F. Pernuš, Comparative evaluation of retrospective shading correction methods. J. Microsc. (Oxford, UK) 208(3), 212-223 (2002)

11. B. Likar, J.A. Maintz, M.A. Viergever, F. Pernuš, Retrospective shading correction based on entropy minimization. J. Microsc. 197(3), 285-295 (2000) 